\title{
NANO-MANAGEMENT OF LINEAR PROPERTIES OF PHOTONIC CRYSTAL FIBERS
}

The array of nanometer air holes positioned in the core can significantly modify the properties of guided mode in photonic crystal fiber. Changing geometrical properties of nanometer holes enables tuning of fiber dispersion and the effective mode area. This is related to losses of mode. In this paper, a rigorous analysis of dispersion and losses has been carried out for photonic crystal fiber with special arrangement of nanometer air holes in the core.

Keywords: Photonic crystal fiber, nanometer air holes ring, dispersion, losses.

\section{Introduction}

The ability of controlling wave propagation has always been a challenge in huge number of applications. Development in this area experienced great progress by revealing the principles of light behavior in photonic crystals [1]. These principles were also applied on waveguides [2 and 3]. Unique properties of these waveguides called photonic crystal fibers (PCFs), are achieved by different methods, but mostly by changing geometrical arrangement (for example modifying the elliptical shape of holes, gradually changing size of holes) or chemical composition of fibers [4 - 6]. Special way of controlling fiber properties is the inclusion and the modification of cylindrical defects with cross-sectional dimensions of tens to hundreds of nanometers within the core. So far this method addressed several groups [7 - 9]. The implementation of one central circular hole with a diameter of approximately $500 \mathrm{~nm}$ the ultra-flat behavior of dispersion with the variation of $2 \mathrm{ps}^{-\mathrm{nm}^{-1}}$. $\mathrm{km}^{-1}$, in the wavelength range of light $700 \mathrm{~nm}$ [8] was verified with simulation methods. Produced fiber with such a structure can be found in [9]. Also influence of hexagonal structure of nanometer air holes in core was shown in [10] to be effective for fine tuning of dispersion, nonlinearity and gain. Effective mode area, in relation to fiber dimension, describes degree of localization of mode in core of fiber. While fibers with large mode area (LMA) are designed for linear performance, in those with small mode area one can expect strong effects of nonlinearities. Combining strong mode confinement within a very small area and the appropriate value of dispersion at wavelength of emitting light source can create conditions for supercontinuum generation [11].

The aim of this paper is to provide deep analysis of the effects of changing geometric parameters of air nanostructures within the core of pure-silica PCF, a focus on linear properties of such PCFs, namely the dispersion and losses.

\section{Geometry of the studied structure and modal analysis}

We use the five-ring-hexagonal-lattice structure of photonic crystal fiber shown in Fig. 1a. The pitch $\Lambda$ of the fiber is $2 \mathrm{~mm}$, $d / \Lambda$ is 0.6 , where $d$ is the diameter of cladding hole, and the effective mode area is $5.7678 \mathrm{~mm}^{2}$. To the area of the solid core formed by omission of the single central hole we add the ring of air nanostructure holes (Fig. 1d, Fig. 1e). The arrangement of ring holes is defined by its radius $L_{\mathrm{n}}$ and nanohole diameter $d_{\mathrm{n}}$.

As it can be seen from Fig. 1 the area of fundamental mode in fiber with nanostructure (e) is slightly compressed (94.58\%) compared to the fiber without nanostructure, but still more than in (d) structure (the effective mode area compressed to $98.79 \%$ ). With both enlargement and the proximity to the innermost cladding ring the nanoholes take over the mode-confining function. Further, to see only the influence of the position we kept the nanohole diameter constant. The mode power distributions with different $L_{\mathrm{n}}$ swept for multiple wavelengths help us visualize the wavelength-selective localization of mode power within the nanostructure (Fig. 2).

\footnotetext{
* Daniel Kacik, Peter Tatar, David Liachovicky

Department of Physics, University of Zilina, Slovakia

E-mail: daniel.kacik@fel.uniza.sk
} 
COMMNICOIIONS

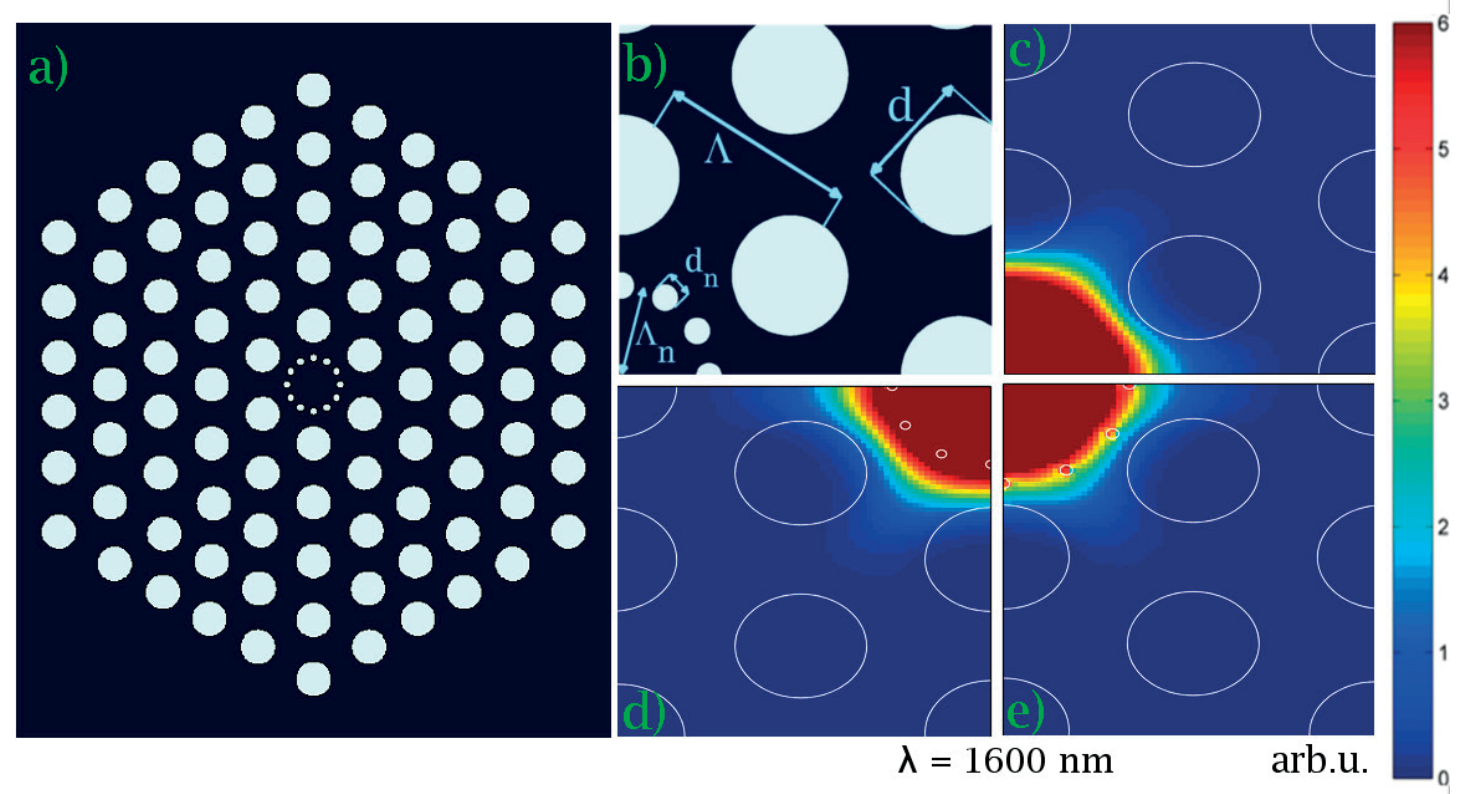

Fig. 1 Structure of the modeled PCF with solid core (a) and details of the structure (b) and of mode distributions of $z$ - component of Poynting vector for PCF (shown $\pi / 2$ quadrant) with $\Lambda=2 m m$ and $d / \Lambda=0.6$ : (c) without nanostructure, (d) with nanostructure where $L_{n}=900 \mathrm{~nm}, d_{n}=90 \mathrm{~nm},(e)$ with nanostructure where $L_{n}=1150 \mathrm{~nm}, d_{n}=115 \mathrm{~nm}$

\begin{tabular}{|c|c|c|c|}
\hline$\lambda$ & without nanostructure & $\Lambda_{\mathrm{n}}=1000 \mathrm{~nm}, d_{\mathrm{n}}=200 \mathrm{~nm}$ & $\Lambda_{\mathrm{n}}=900 \mathrm{~nm}, d_{\mathrm{n}}=200 \mathrm{~nm}$ \\
\hline $420 \mathrm{~nm}$ & & & \\
\hline \multicolumn{4}{|l|}{$660 \mathrm{~nm}$} \\
\hline \multicolumn{4}{|l|}{$920 \mathrm{~nm}$} \\
\hline $1140 \mathrm{~nm}$ & & & \\
\hline
\end{tabular}




(1400nm

Fig. 2 Mode distributions of z-component of Poynting vector for PCFs with constant $d_{n}=200 \mathrm{~nm}$, but radius of rings $L_{n}=1000 \mathrm{~nm}$ and $900 \mathrm{~nm}$ for different wavelengths of light.

\section{Propagation characteristic: Results and discussion}

To investigate the sensitivity of linear properties of PCF to the nanostructure with respect to the position in the core area (at different distance from the center) the nanostructure ring was scaled with constant ratio $d_{\mathrm{n}} / L_{\mathrm{n}}=0.1$ which is small relative to the wavelength. In Fig. 3 we observe only slight changes in dispersion values that retain the shape of the dispersion curve of fiber without nanostructure ring in core. The small holes can be perceived as elements that lower the overall refractive index of the homogeneous core. In accordance with this conception is the greatest decrease of dispersion curve associated with the largest change in the waveguide geometry (structure given at Fig. 1e: $d_{\mathrm{n}}=115 \mathrm{~nm}$ ). For this configuration of holes, which are nearly adjacent to the innermost cladding ring, the loss curve does not obey monotonic increase with nanohole diameter coupled with increased radius of the ring nanostructure.

In Fig. 4 we see that concentrating the ring by changing $L_{\mathrm{n}}$ with $100 \mathrm{~nm}$ step causes significant red-shift $(200 \mathrm{~nm})$ of lower zero dispersion wavelength (ZDW) and smaller blue-shift (100 $\mathrm{nm}$ ) of higher ZDW. Although such change does not yield any profile that would not be achievable by cladding modification, it is manifestation of sensitivity to the density and thickness of the formed ring. If we continue to decrease $L_{\mathrm{n}}$ with constant hole diameter, ring closure is stronger and also mode power distribution slowly transforms into a shape resembling more an annulus. Nanoholes touch at $d_{\mathrm{n}} / L_{\mathrm{n}}=0.515$, which is certainly not a manufacturable case of fiber.

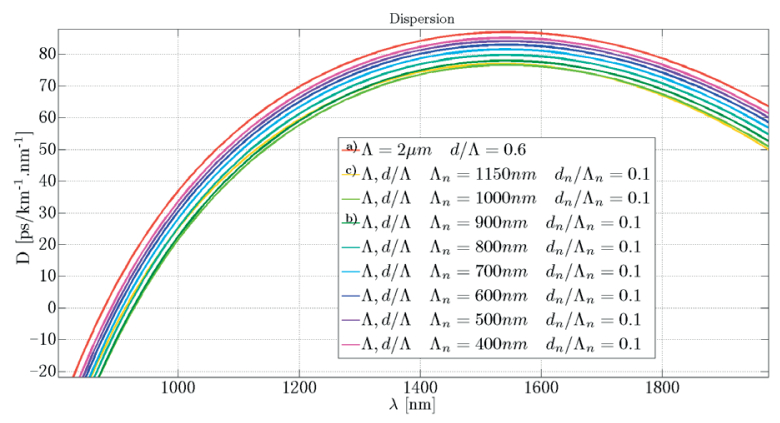

a)
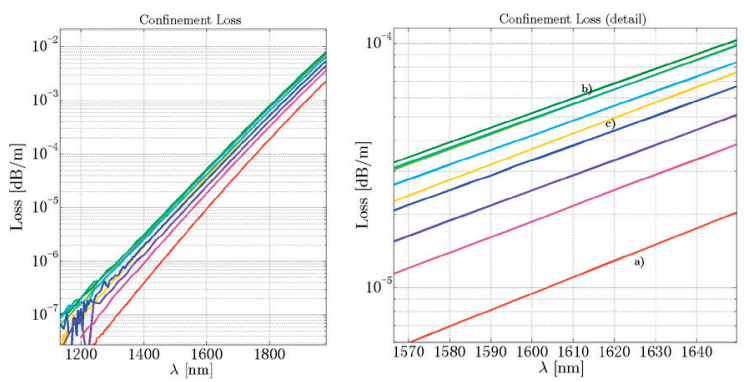

b)

Fig. 3 Dispersion (a) and confinement losses (b) for different radii of ring nanostructures and constant relative nanohole size (structures from Fig. 1b) 

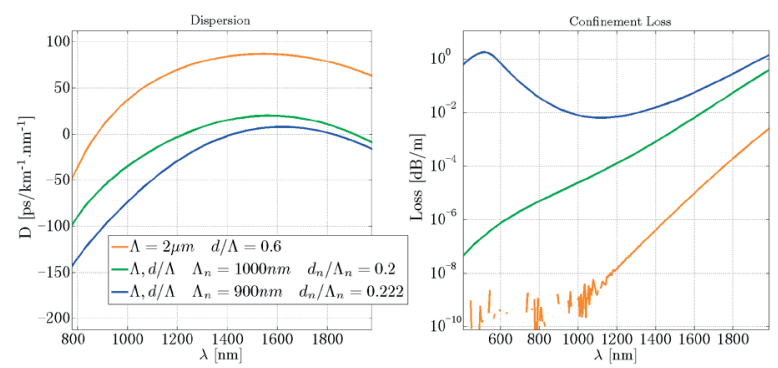

Fig. 4 Dispersion and confinement losses for different radii of ring nanostructures and the same diameter of nanoholes $(200 \mathrm{~nm})$

(structures from Fig. 2cde)

Noteworthy is the loss plot of the structure $L_{\mathrm{n}}=900 \mathrm{~nm}$ and $d_{\mathrm{n}} / L_{\mathrm{n}}=0.222$ (Fig. 4): such fiber experiences region of stronger confinement in near-infrared while the losses at the blue-green part of the studied spectrum exceed $1 \mathrm{~dB} / \mathrm{m}$.

Such behavior corresponds with mode power distributions illustrated in Fig. 2. The fundamental mode of the structure $\left(L_{\mathrm{n}}=\right.$ $900 \mathrm{~nm}, d_{\mathrm{n}}=200 \mathrm{~nm}$ ) propagates partially in the cladding more than in the other structure $\left(L_{\mathrm{n}}=1000 \mathrm{~nm}, d_{\mathrm{n}}=200 \mathrm{~nm}\right)$. At wavelength $420 \mathrm{~nm}$ the power surrounds the nanoholes without penetrating inside. We can surmise that nanoring enhances the light confinement in the way similar to PCF rings. With increasing wavelength the power expands through the nanoring and at $\lambda \approx 1200 \mathrm{~nm}$ resembles the mode of the structure with $L_{\mathrm{n}}=1000$ $\mathrm{nm}$. Towards longer wavelengths both structures leak the power into the cladding which corresponds to the loss curves in Fig. 4 running in quasi-parallel manner at that spectral region.

Simulations were carried out for smaller $L_{\mathrm{n}}$ and large relative hole size $d_{\mathrm{n}} / L_{\mathrm{n}}=0.48 \pm 0.05$, too. Qualitatively, in Fig. 5 for small nanoholes arranged in the vicinity of the center the scenario is similar to that in Fig. 3. With the expansion of the nanoring the dispersion curve submerges and the structure allows more leakage. The window of anomalous dispersion is being contracted and shifted more to the shorter wavelengths region.
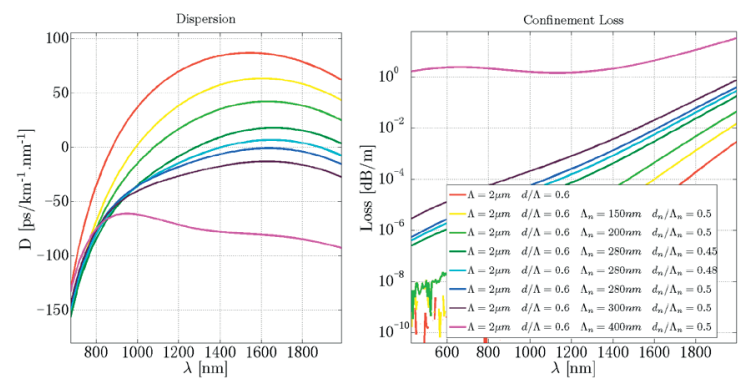

Fig. 5 Dispersion (a) and confinement losses (b) for smaller radii of ring nanostructures and constant relative nanohole size

Quantitatively, the level of dispersion drops very rapidly. A particular structure around $\left(L_{\mathrm{n}} \approx 280 \mathrm{~nm}\right.$ and $\left.d_{\mathrm{n}} / L_{\mathrm{n}} \approx 0.5\right)$ exhibits only one ZDW. Further increasing of the nanoring radius yields all-normal dispersion profiles.

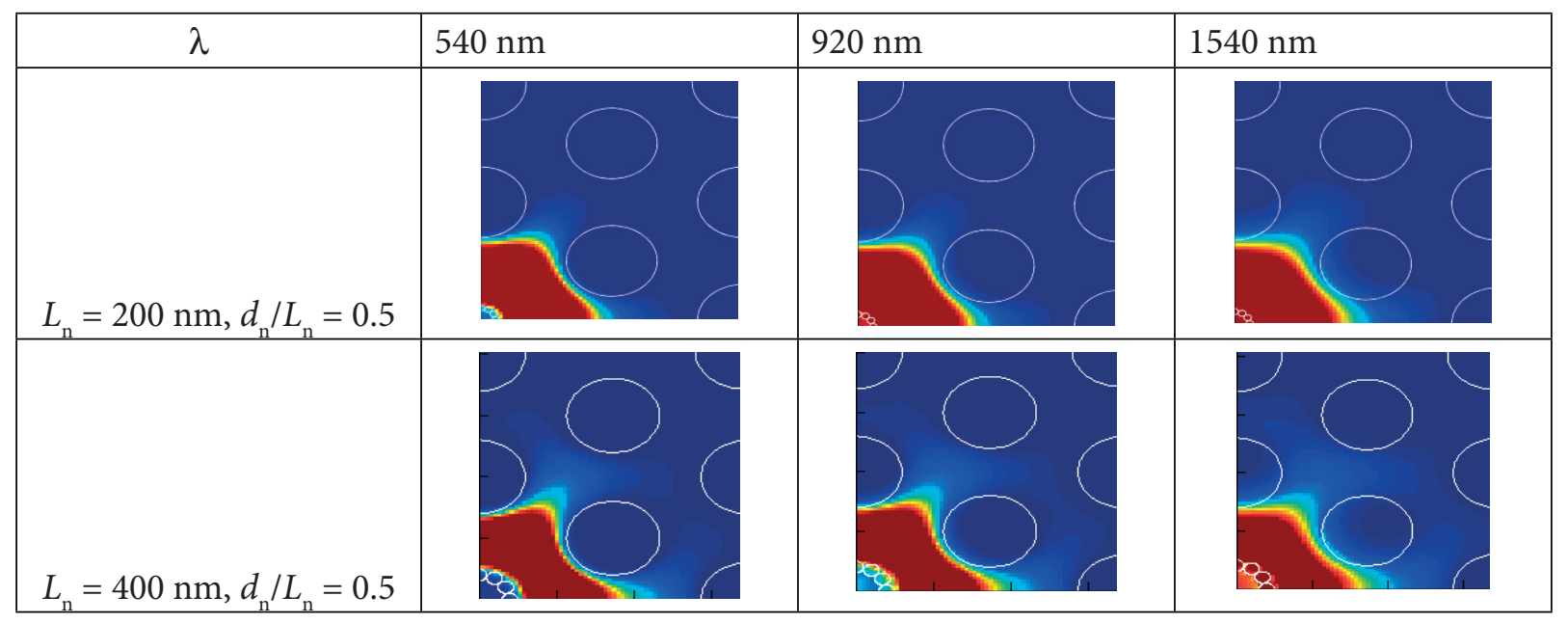

Fig. 6 Mode distributions of z-component of Poynting vector for PCF with constant $d / L_{n}=0.5$, but for different radii of ring nanostructure; penetration of light through the ring occurs for different structures at different wavelength 
For the design of fiber having only one ZDW we separate the influence of holes' size. The difference between structures with $L_{n}$ $=280 \mathrm{~nm}$ is in the hole size: $140 \mathrm{~nm}, 134 \mathrm{~nm}$ and $126 \mathrm{~nm}$ (see legend in Fig. 5). For example, at $1650 \mathrm{~nm}$ the change of the hole diameter as small as $6 \mathrm{~nm}$ will induce relatively strong dispersion change $6.8 \mathrm{ps} . \mathrm{nm}^{-1} . \mathrm{km}^{-1}$ and if we again diminish the diameter $(8$ $\mathrm{nm}$ ) this change will result in $11.2 \mathrm{ps} . \mathrm{nm}^{-1} \cdot \mathrm{km}^{-1}$.

With increasing $L_{\mathrm{n}}$ the sensitivity of dispersion does not rise dramatically, rather we notice significant changes in loss curves. Positioning larger nanoholes roughly to the half way from fiber center to first cladding ring prevents the fundamental mode from the low-loss guidance around the middle of the studied spectrum where can be also substantial mode leakage (see Fig. 6).

For power distribution of modes, the ring is closed and at short wavelengths prevents the power to be localized within such small area. With increasing wavelength the power starts to penetrate into the area encircled by nanoholes and finally at longer wavelengths the power of mode is comparable inside and outside the nanoring. The wavelength at which the light starts to disobey the nanoring as an obstacle depends on its radius. The smaller the ring, the shorter is this wavelength, which is illustrated in Fig. 6.

So far the effects of nanostructure were examined in terms of its distance from center and first inner ring of cladding. Last modification taken into account is how it behaves with holes having same position, but difference size.

Fig. 7 illustrates the nanostructures within core and one can see that, in fact, they form the core, becoming the innermost ring, since their effective mode areas are smaller than those of the core without nanostructure (data not shown). For larger hole size (Figs. 7c, 7d) at longer wavelengths they release the strong mode confinement thus extending the effective mode areas over those of structures with smaller nanoholes (Figs. 7a, 7b).

Concerning the dispersion, the red shift of ZDW is characteristic for the small holes whilst for the large ones strong waveguide contribution to anomalous dispersion shifts the ZDW towards blue: ZDW is $817 \mathrm{~nm}$ and $738 \mathrm{~nm}$ for the fibers given by Figs. $7 \mathrm{c}$ and $7 \mathrm{~d}$, respectively. In this case, the large holes of diameter $d_{\mathrm{n}}>\lambda / 2$ provoke strong oscillations of the dispersion curve. At near infrared wavelengths the slope of the strong anomalous dispersion is crossing zero and the curve falls to the

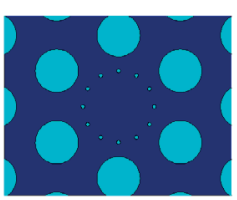

a)

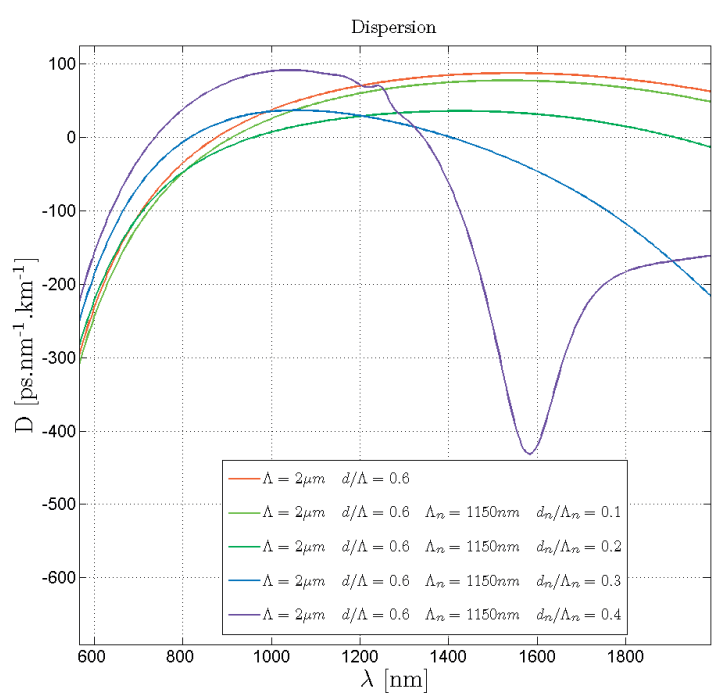

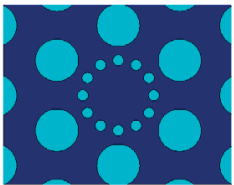

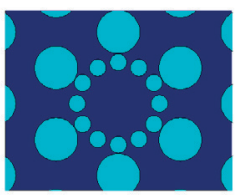

c)

d)

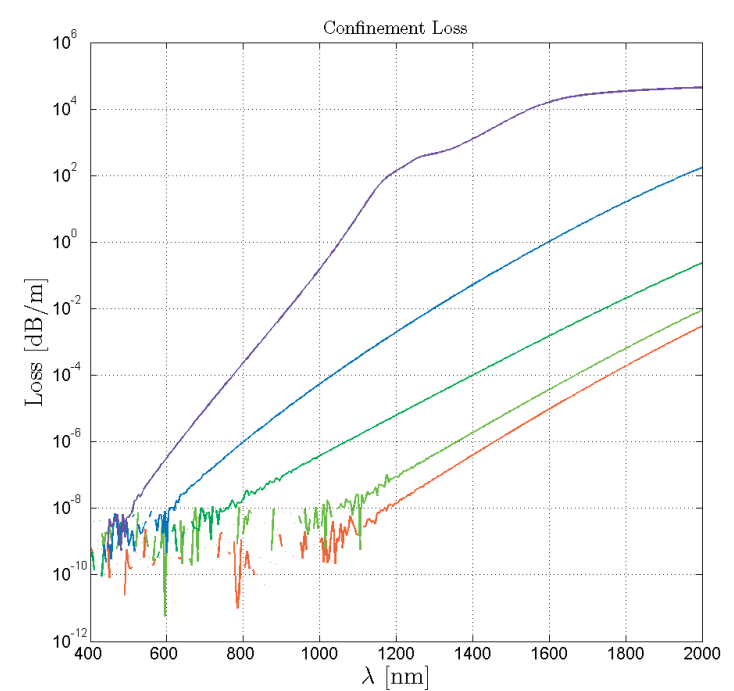

e)

Fig. 7 Ring nanostructure with holes (a) $115 \mathrm{~nm}$, (b) $174 \mathrm{~nm}$, (c) $261 \mathrm{~nm}$, (d) $348 \mathrm{~nm}$, all $1150 \mathrm{~nm}$ far from the center, (e) dispersion and losses as a function of wavelength for the structures $(a)(b)(c)(d)$ 
normal dispersion half plane, which is connected with steep grow of loss.

Finally, the large normal dispersion region in mid infrared band with remarkable dip corresponds to the modes with effective mode area larger than $8 \mathrm{~mm}^{2}$ which visually means radius of the circle circumscribing such effective mode area is app. 300 $\mathrm{nm}^{2}$ greater than that in case of core without nanostructure. At $\lambda \approx 1900 \mathrm{~nm}$ the mode power, however, leaks strongly into the cladding.

\section{Conclusion}

We carried out detailed analysis of the dispersion, losses and cross-sectional mode power distribution of photonic crystal fibers with nanohole ring structure placed in the core. The simulations revealed a great sensitivity of these properties to changes of geometrical parameters of nanostructure involving position and size of arrayed nanoholes. With proper adjustment of the available parameters the desired dispersion characteristic could be achieved. Strong ties between wild dispersion profiles and high losses were manifested in critically leaky structures.

Furthermore, due to their circular arrangement the nanoholes do not perfectly fit the pattern of cladding holes and thus not serve as an extension of periodical cladding, which significantly affects the mode shape (for nanoholes' size further above homogenization limit).

Besides, it should be noted that whether the structure is producible or not is questionable since the holes can be too small and close to each other to not collapse. Supposedly, the silica-air combination would make the fabrication of such fibers even more challenging issue.

\section{Acknowledgements:}

The authors would like to express their thanks to Dr. Peter Tvarožek for his idea and helpfull discussion. This work was partly supported by the Slovak National Grant Agency No. VEGA 1/1058/11 and No. VEGA 1/0528/12.

\section{References:}

[1] Johnnopoulos, J. D., Johnson, S. G., WINN, J. N., MEADE, R. D.: Photonic Crystals: Molding the Flow of Light, Princeton University Press, 2008.

[2] KNIGHT, J. C.: Photonic Crystal Fibers, Nature 424, 8471, 2003.

[3] RUSSEL, P. St. J.: Photonic Crystal Fibers, J. of Lightwave Technology 24, 4729, 2006.

[4] TARTARINI, G., PANSERA, M., ALKESKJOLD, T. T., BJARKLEV, A., BASSI, P.: Polarization Properties of Elliptical-Hole Liquid Crystal Photonic Bandgap Fibers, J. Lightwave Technol. 25, pp. 2522-2530, 2007.

[5] ReNVERSEZ, G., KUHLMEY, B., MCPHEDRAN, R.: Dispersion Management with Microstructured Optical Fibers: Ultraflattened Chromatic Dispersion with Low Losses, Opt. Lett. 28, pp. 989-991, 2003.

[6] KANKA, J.: Design of Photonic Crystal Fibers with Highly Nonlinear Glasses for Four-wave-mixing Based Telecom Applications, Opt. Express 16, pp. 20395-20408, 2008.

[7] WIEDERHECKER, G. S., CONDEIRO, C. M. B., COUNY, F., BENABID, F., MAIER, S. A., KNIGHT, J. C., CRUZ, C. H. B., FRAGNITO, H. L.: Field Enhancement within an Optical Fibre with a Subwavelength Air Core, Nature Photonics, 1, pp. 115 - 118 , 2007.

[8] SAITOH, K., FlOROUS, N., KOSHIBA, M.: Ultra-flattened Chromatic Dispersion Controllability Using a Defected-core Photonic Crystal Fiber with Low Confinement Losses, Opt. Express, vol. 13, pp. 8365-8371, 2005.

[9] RUAN, Y., EBENDORFF-HEIDEPRIEM, H., AFSHAR, S.,MONRO, T. M.: Light Confinement within Nanoholes in Nanostructured Optical Fibers, Opt. Express, vol. 18, pp. 26018-26026, 2010.

[10] SEREBRYANNIKOV E. E., ZHELTIKOV, A. M.,: Nanomanagement of Dispersion, Nonlinearity, and Gain of Photonic-crystal Fibers: Qualitative Arguments of the Gaussian-mode Theory and Nonperturbative Numerical Analysis, J. Opt. Soc. Am. B, vol. 23, pp. 1700-1707, 2006 .

[11] SHAVRIN, I., TVAROZEK, P., KOYS, M., SCHUSTER, K., NOVOTNY, S., LUDVIGSEN, H.: EXTREMELY WHITE SUPERCONTINUUM GENERATION IN THREE-HOLE SUSPENDED-CORE FIBER, Conference on Lasers and ElectroOptics Europe and 12th European Quantum Electronics Conference, CLEO EUROPE/EQEC 2011, art. No. 5943001, 2011. 